\title{
Interactive comment on "Meteorological and evaluation datasets for snow modelling at ten reference sites: description of in situ and bias-corrected reanalysis data" by Cécile B. Menard et al.
}

Anonymous Referee \#1

Received and published: 15 April 2019

The paper entitled "Meteorological and evaluation datasets for snow modelling at ten reference sites: description of in situ and bias-corrected reanalysis data" is an overall description of ten datasets that have been somewhat standardized to ease their use as benchmarks for model developments. The work undertaken by the authors is of crucial importance to the scientific community and this paper will certainly help future users to better understand how to use these datasets. The discussion is particularly well written and enlightening. Section 2 needs a bit of work in terms of flow of ideas, as it appears to have been written by multiple authors with abrupt (and sometimes 
confusing) transitions between description elements.

I therefore recommend this paper for minor revisions.

ESSDD

Major comments:

Section 2 seems to be written by multiple authors and is difficult to read due to abrupt transitions. As an example, the sentence starting on line 128 should be the beginning of a new paragraph, as the reader may wonder if that sentence refers to Sodankyla only.

Line 202: add a note that the peculiar behavior seen in the SAP site will be discussed later on, or better yet, give the reader a quick explanation.

Lines 207-210: I don't think that differences between annual snowfall and peak SWE should be termed discrepancies. A number of processes can lead to these differences, such as sublimation, rain-on-snow events, interception, melting events, etc., all of which are natural and not erroneous as the word "discrepancies" implies. I certainly would not expect a perfect match between annual snowfall and peak SWE.

Section 2.1.5: reading the text, it is not clear whether the datasets provide relative humidity, specific humidity, or both (which would be best, as it would allow users to choose). Please clarify.

Line 339: What type of bias corrections do you refer to? Is it what is described in the paragraph starting on line 358? If so, please mention it, otherwise please elaborate.

Line 293-294: Is the rejection applied to all sites? Please clarify.

Minor comments:

Line 190: underestimated

Line 274: Please add the table number.

Line 380: Please add table number. 
Table 3: Third column, replace Snow by snow. Last column, what is the number 670 referring to?

ESSDD

Interactive comment on Earth Syst. Sci. Data Discuss., https://doi.org/10.5194/essd-2019-12, 2019.

Interactive

comment 